University of Shizuoka

US-97-05

July 1997

\title{
Neutrino Masses and Mixings in a Universal Seesaw Mass Matrix Model
}

\author{
Yoshio Koide \\ Department of Physics, University of Shizuoka \\ 395 Yada, Shizuoka 422, Japan
}

\begin{abstract}
Neutrino masses and mixings are investigated on the basis of a universal seesaw mass matrix model, in which quark (except for top) and charged lepton mass matrices $M_{f}$ and neutrino mass matrix $M_{\nu}$ are given by $M_{f} \simeq m_{L} M_{F}^{-1} m_{R}$ and $M_{\nu} \simeq m_{L} M_{F}^{-1} m_{L}^{T}(F=N)$, respectively. For a simple model which can successfully describe quark masses and mixings, we find that the observed neutrino data (except for the solar neutrino data) are favor to the intermediate mass scales $O\left(m_{R}\right)=10^{11} \mathrm{GeV}$ and $O\left(M_{F}\right)=10^{13} \mathrm{GeV}$ together with $O\left(m_{L}\right)=10^{2}$ $\mathrm{GeV}$. In spite of the largesse of $O\left(m_{R}\right)$, the observed top quark mass can be consistently understood from the would-be seesaw mass matrix with these mass scales.
\end{abstract}

PACS numbers: 14.60.Pq, 12.15.Ff, 12.60.-i, 96.40.Tv

* E-mail: koide@u-shizuoka-ken.ac.jp 


\section{Introduction}

One of the most exciting problem in the quark and lepton physics is to give a natural understanding of the observed hierarchical mass spectra of quarks and leptons in relation to the electroweak scale $\Lambda_{W}$. In the conventional models, the fundamental fermions acquire their masses through the symmetry breaking of the electroweak symmetry at the energy scale $\mu=\left\langle\phi_{L}^{0}\right\rangle \equiv \Lambda_{W}=174 \mathrm{GeV}$. However, the observed mass values except for the top quark mass $m_{t}$ are considerably small compared with $\Lambda_{W}$. Especially, the neutrino masses are invisibly small (or exactly zero). In relation to this problem, we know an interesting mechanism, the so-called "seesaw" mechanism.

The mechanism [1] was first proposed in order to answer the question why neutrino masses are so invisibly small: $M_{\nu} \simeq-m M^{-1} m^{T}$, where $m$ is a Dirac mass matrix with the order of the conventional quark and charged lepton masses and $M$ is a Majorana mass matrix of the right-handed neutrino $\nu_{R i}(i$ is family-index). If we suppose $m_{\nu} \leq 10 \mathrm{eV}$ and $m=O\left(m_{\tau}\right)$, the Majorana mass $M$ must be larger than $10^{8} \mathrm{GeV}$. Usually, in the most models, the order of $M$ is taken as the order of the unification energy scale.

On the other hand, in order to understand why quark masses are so small compared with the electroweak scale $\Lambda_{W}$, the mechanism was applied to the quarks [2]: $M_{f} \simeq-m_{L} M_{F}^{-1} m_{R}$, where $M_{F}$ is a mass matrix of hypothetical heavy fermions $F_{i}$. If we take $m_{L} \sim m_{W} \sim 10^{2} \mathrm{GeV}, m_{R} \sim 10^{3} \mathrm{GeV}$ and $m_{f} \sim$ a few $\mathrm{GeV}$, then the heavy fermion mass $M_{F}$ must be of the order of $10^{5} \mathrm{GeV}$. However, it seems to be not economical that we have two different mass scales $M$ and $M_{F}$. Can we build a model with the same mass scale for $M$ and $M_{F}$ ? The answer is Yes. Since the neutrino and quark mass matrices $M_{\nu}$ and $M_{f}$ are given by $M_{\nu} \simeq-m_{L} M_{F}^{-1} m_{L}^{T}$ $(F=N)$ and $M_{f} \simeq-m_{L} M_{F}^{-1} m_{R}(F=U, D)$, respectively, we can understand the smallness of the neutrino masses by assuming $O\left(m_{L}\right) / O\left(m_{R}\right)=O\left(m_{\nu} / m_{f}\right)$ $(f=u, d)[3]$.

By the way, for the seesaw model for quarks, there seems to be a stumbling block: it seems that the observed top quark mass [4] $m_{t} \sim \Lambda_{W}$ apparently takes objection to the application of the seesaw mass matrix model to quarks. However, recently, it has been pointed out $[5,6]$ that the seesaw mass matrix model for quarks is rather preferable to understand why only top quark mass $m_{t}$ is of the order of the electroweak scale $\Lambda_{W}$ and why $m_{t}$ is so singularly enhanced in the third-family in contrast with $m_{u} \sim m_{d}$ in the first family. In the framework of the $\mathrm{SU}(2)_{L} \times \mathrm{SU}(2)_{R} \times \mathrm{U}(1)_{Y}$ gauge model, the $6 \times 6$ would-be seesaw mass matrix for 
the fermion $(f, F)$ is given by

$$
M=\left(\begin{array}{cc}
0 & m_{L} \\
m_{R} & M_{F}
\end{array}\right)=m_{0}\left(\begin{array}{cc}
0 & Z_{L} \\
\kappa Z_{R} & \lambda Y_{f}
\end{array}\right),
$$

where $f_{i}(i=1,2,3$ : family index) are the ordinary quarks $(f=u, d)$ and leptons $(f=\nu, e)$, and $F_{i}$ are hypothetical vector-like heavy fermions corresponding to $f_{i}$. The fermions $f_{i}$ and $F_{i}$ belong to $f_{L}=(2,1), f_{R}=(1,2), F_{L}=(1,1)$ and $F_{R}=(1,1)$ of $\mathrm{SU}(2)_{L} \times \mathrm{SU}(2)_{R}$, respectively. The symmetries $\mathrm{SU}(2)_{L}$ and $\mathrm{SU}(2)_{R}$ are broken by the vacuum expectation values of the Higgs scalars $\phi_{L}$ and $\phi_{R}$, i.e., the mass matrices $m_{L}$ and $m_{R}$, respectively. For convenience, in (1.1), we have denoted the matrices $m_{L}, m_{R}$ and $M_{F}$ in terms of the matrices $Z_{L}, Z_{R}$ and $Y_{f}$, which are of the order of one, i.e., $\operatorname{Tr}\left(Z_{L} Z_{L}^{\dagger}\right)=O(1), \operatorname{Tr}\left(Z_{R} Z_{R}^{\dagger}\right)=O(1)$ and $\operatorname{Tr}\left(Y_{f} Y_{f}^{\dagger}\right)=O(1)$. It is well-known that the mass matrix (1.1) leads to the seesaw expression

$$
M_{f} \simeq-m_{L} M_{F}^{-1} m_{R},
$$

for the case $|\lambda| \gg|\kappa| \gg 1$ and $\operatorname{det} M_{F} \neq 0$. On the contrary, for the case $\operatorname{det} M_{F}=$ 0 , the mass spectrum is given by $[5,6]$

$$
\begin{aligned}
m_{1}, m_{2} & \sim(\kappa / \lambda) m_{0} & , \\
m_{3} & \sim m_{0} & =O\left(m_{L}\right), \\
m_{4} & \sim \kappa m_{0} & =O\left(m_{R}\right), \\
m_{5}, m_{6} & \sim \lambda m_{0} & =O\left(M_{F}\right),
\end{aligned}
$$

independently of the explicit structures of $Z_{L}, Z_{R}$ and $Y_{f}$. Note that the third fermion mass $m_{3}^{f}$ is given by $m_{3}^{f} \sim m_{0}$ without the suppression factor $\kappa / \lambda$. Therefore, if we build a model such as $\operatorname{det} M_{F}=0$ in up-quark sector, we can understand why top-quark alone has a mass of the order of $m_{L}\left(\sim \Lambda_{W}\right)$. This fact (1.3) was first explicitly demonstrated by Fusaoka and the author [5] on the basis of a special seesaw mass matrix model, where $M_{F}$ is given by the form [(unit matrix)+(a rank-one matrix)], and then re-stressed by Morozumi et al. [6], on the basis of a general study.

Thus, the result (1.3) seems to support the idea that the seesaw mass matrix model for the quarks should be taken seriously. Then, the problem is rather in the neutrino sector: Can the seesaw model, which can successfully describe the quark masses and mixings, satisfactorily describe the neutrino masses and mixings, too? The purpose of the present paper is to discuss the neutrino phenomenology on the 
bases of an explicit model which can satisfactorily describe the quark masses and mixings.

In the next section, we discuss the $12 \times 12$ mass matrix of the twelve Majorana neutrinos and we will re-derive the well-known form $M_{\nu} \simeq-\left(m_{0} / \lambda\right) Z_{L} Y_{\nu}^{-1} Z_{L}^{T}$ for the conventional light neutrinos. In Sec. III, we introduce a model (Model I) with $Z_{L}=Z_{R}$ and $Y_{\nu}=[($ unit matrix $)+($ rank-one matrix $)]$ as a simple example of the matrices $Z_{L}, Z_{R}$ and $Y_{F}$. In Sec. IV, we try to explain all of the present neutrino data, the solar, atmospheric and LSND neutrino data, by Model I. However, we will fail in the simultaneous explanation of the three data, and we can give a satisfactory explanation only by giving up the explanation of one of the three. For example, if we give up an explanation of the solar neutrino data, we can give the reasonable values of $\left(\Delta m^{2}, \sin ^{2} 2 \theta\right)$ for the atmospheric and LSND neutrinos, and we obtain the intermediate mass scales $\kappa m_{0} \sim 10^{11} \mathrm{GeV}$ and $\lambda m_{0} \sim 10^{13} \mathrm{GeV}$ together with $m_{0}=312 \mathrm{GeV}$. In spite of the largeness of $O\left(m_{R}\right)$, the model will be able to give a reasonable value of the top quark mass $m_{t}$ (and, of course, reasonable other quark masses and mixings). Finally, Sec. $\mathrm{V}$ is devoted to the conclusions and remarks.

\section{Neutrino Mass Matrix}

The neutral lepton mass matrix which is sandwiched between $\left(\bar{\nu}_{L}, \bar{\nu}_{R}^{c}, \bar{N}_{L}, \bar{N}_{R}^{c}\right)$ and $\left(\nu_{L}^{c}, \nu_{R}, N_{L}^{c}, N_{R}\right)^{T}$, where $\nu_{L}^{c} \equiv\left(\nu_{L}\right)^{c} \equiv C \bar{\nu}_{L}^{T}$ and so on, is given by

$$
M=\left(\begin{array}{cccc}
0 & 0 & 0 & m_{L} \\
0 & 0 & m_{R}^{T} & 0 \\
0 & m_{R} & M_{M} & M_{D} \\
m_{L}^{T} & 0 & M_{D}^{T} & M_{M}
\end{array}\right)
$$

where $M_{D}\left(\equiv M_{N}\right)$ and $M_{M}$ are Dirac and Majorana mass matrices of the neutral heavy fermions $N_{i}$. The matrices $m_{L}$ and $m_{R}$ are universal for all fermion sectors, i.e., $f=u, d, e, \nu$, and the differences among up-/down- quark/lepton masses are generated by the differences of $M_{F} \equiv \lambda Y_{f}$. The heavy fermions $F_{i}$ belong to $(1,1)$ of $\mathrm{SU}(2)_{L} \times \mathrm{U}(2)_{R}$. Besides, the neutral heavy leptons $N_{i}$ do not have the $\mathrm{U}(1)$ charge. Therefore, it is likely that when the Dirac masses $\left(M_{D}\right)_{i j}$ are generated between $\bar{N}_{L i}$ and $N_{R j}$, the Majorana masses $\left(M_{M}\right)_{i j}$ are also generated between $\bar{N}_{L i}$ and $N_{L j}^{c}\left(\bar{N}_{R i}^{c}\right.$ and $\left.N_{R j}\right)$ with the same structure at the same energy scale $\mu=\lambda m_{0}$. Hereafter, we put $M_{M}=M_{D} \equiv M_{N} \equiv \lambda m_{0} Y_{\nu}$.

For the case $M_{M}=M_{D}$, the diagonalization must be done carefully because the determinant of the $6 \times 6$ sub-matrix for $\left(N_{L}^{c}, N_{R}\right)$ in the $12 \times 12$ matrix $(2.1)$ 
becomes zero. First, we rotate the matrix $(2.1)$ on the $\left(N_{L}^{c}, N_{R}\right)$-plane by the angle $\pi / 4$. Then, the mass matrix (2.1) becomes

$$
M^{\prime}=\frac{m_{0}}{\sqrt{2}}\left(\begin{array}{cccc}
0 & 0 & -Z_{L} & Z_{L} \\
0 & 0 & \kappa Z_{R}^{T} & \kappa Z_{R}^{T} \\
-Z_{L}^{T} & \kappa Z_{R} & 0 & 0 \\
Z_{L}^{T} & \kappa Z_{R} & 0 & 2 \sqrt{2} \lambda Y_{\nu}
\end{array}\right)
$$

We can see that, of the twelve components of the neutrinos, the three are approximately described by the mass matrix $2 \lambda m_{0} Y_{\nu}$. The remaining $9 \times 9$ mass matrix is given by

$$
M^{\prime} \simeq \frac{m_{0}}{\sqrt{2}}\left(\begin{array}{ccc}
0 & 0 & -Z_{L} \\
0 & 0 & \kappa Z_{R}^{T} \\
-Z_{L}^{T} & \kappa Z_{R} & 0
\end{array}\right)-\frac{m_{0}}{4 \lambda}\left(\begin{array}{ccc}
Z_{L} Y^{-1} Z_{L}^{T} & \kappa Z_{L} Y^{-1} Z_{R} & 0 \\
\kappa Z_{R}^{T} Y^{-1} Z_{L}^{T} & \kappa Z_{R}^{T} Y^{-1} Z_{R} & 0 \\
0 & 0 & 0
\end{array}\right)
$$

where we have used the formula of the seesaw approximation for the $(n+m) \times(n+m)$ matrix $M$ :

$$
M=\left(\begin{array}{cc}
A & B \\
C & D
\end{array}\right) \Longrightarrow M^{\prime} \simeq\left(\begin{array}{cc}
A-B D^{-1} C & 0 \\
0 & D
\end{array}\right)
$$

By using

$$
R \simeq\left(\begin{array}{ccc}
1 & \varepsilon & 0 \\
-\varepsilon^{\dagger} & 1 & 0 \\
0 & 0 & 1
\end{array}\right), \quad \varepsilon=\frac{1}{\kappa} Z_{L}\left(Z_{R}^{T}\right)^{-1}
$$

the matrix $(2.3)$ is transformed into

$$
R M^{\prime} R^{T} \simeq m_{0}\left(\begin{array}{ccc}
-\frac{1}{\lambda} Z_{L} Y^{-1} Z_{L}^{T} & -\frac{\kappa}{2 \lambda} Z_{L} Y^{-1} Z_{R} & 0 \\
-\frac{\kappa}{2 \lambda} Z_{R}^{T} Y^{-1} Z_{L} & -\frac{\kappa^{2}}{4 \lambda} Z_{R}^{T} Y^{-1} Z_{R} & \frac{\kappa}{\sqrt{2}} Z_{R} \\
0 & \frac{\kappa}{\sqrt{2}} Z_{R} & 0
\end{array}\right)
$$


Therefore, we obtain the following twelve Majorana neutrinos: (i) three heavy Majorana neutrinos with masses of the order of $\lambda m_{0}$, whose mass matrix is approximately given by

$$
M_{\text {heavy }} \simeq 2 M_{N}=2 \lambda m_{0} Y_{\nu},
$$

(ii) three sets of almost degenerate two Majorana neutrinos (the pseudo-Dirac neutrino [7]) with masses of the order of $\kappa m_{0}$, whose mass matrix is approximately given by

$$
M_{P S-D} \simeq\left(\begin{array}{cc}
-\frac{1}{4} m_{R}^{T} M_{N}^{-1} m_{R} & \frac{1}{\sqrt{2}} m_{R}^{T} \\
\frac{1}{\sqrt{2}} m_{R} & 0
\end{array}\right)=\kappa m_{0}\left(\begin{array}{cc}
-\frac{\kappa}{4 \lambda} Z_{R}^{T} Y_{\nu}^{-1} Z_{R} & \frac{\kappa}{\sqrt{2}} Z_{R}^{T} \\
\frac{\kappa}{\sqrt{2}} Z_{R} & 0
\end{array}\right)
$$

and (iii) three light Majorana neutrinos with masses of the order of $(1 / \lambda) m_{0}$, whose mass matrix is approximately given by

$$
M_{\nu} \simeq-m_{L} M_{N}^{-1} m_{L}^{T}=-\frac{m_{0}}{\lambda} Z_{L} Y_{\nu}^{-1} Z_{L}^{T} .
$$

Note that, differently from the models by Berezhiani and Davidson-Wari [3], there are no neutrinos with masses of the order of $\left(\kappa^{2} / \lambda\right) m_{0}$, i.e., whose mass matrix is approximately given by $m_{R} M_{N}^{-1} m_{R}^{T}$.

The neutrinos which are described by the mass matrix (2.9) consist of almost left-handed neutrinos $\nu_{L i}$. Therefore, our task is to seek for such matrix forms of $Z_{L}, Z_{R}$ and $Y_{f}$ as those can give reasonable quark and lepton masses, Cabibbo-Kobayashi-Maskawa (CKM) [8] matrix, and neutrino mixings, where the mass matrices of down-quarks and charged leptons are given by (1.2), i.e., $M_{f} \simeq$ $(\kappa / \lambda) Z_{L} Y_{f}^{-1} Z_{R}$ with $\operatorname{det} Y_{f} \neq 0$, that of up-quarks is given by (1.1) with $\operatorname{det} Y_{u}=0$, and that of neutrinos is given by (2.9).

\section{A Simple Case}

As an explicit model of the universal seesaw mass matrix models which give phenomenologically reasonable predictions for quarks, there is a model which has been proposed by Fusaoka and the author [5]. In their model, the matrices are simply taken by

$$
Z_{L}=Z_{R} \equiv Z
$$

and

$$
Y_{f}=\mathbf{1}+3 b_{f} X
$$


where 1 and $X$ are a $3 \times 3$ unit matrix and a rank-one matrix normalized as $X^{2}=X$, respectively. They have assumed that the matrix $Z$ is given by a diagonal form in the family-basis on which $X$ is democratic form:

$$
Z=\left(\begin{array}{ccc}
z_{1} & 0 & 0 \\
0 & z_{2} & 0 \\
0 & 0 & z_{3}
\end{array}\right), \quad X=\frac{1}{3}\left(\begin{array}{ccc}
1 & 1 & 1 \\
1 & 1 & 1 \\
1 & 1 & 1
\end{array}\right)
$$

They have used the input values

$$
\frac{z_{1}}{\sqrt{m_{e}}}=\frac{z_{2}}{\sqrt{m_{\mu}}}=\frac{z_{3}}{\sqrt{m_{\tau}}}=\frac{1}{\sqrt{m_{e}+m_{\mu}+m_{\tau}}}
$$

from $M_{e} \simeq(\kappa / \lambda) m_{0} Z \cdot \mathbf{1} \cdot Z$ by assuming $b_{e}=0$ in the charged lepton sector as a trial. Then, for up-quark sector with $\operatorname{det} Y_{u}=0$, i.e., with $b_{u}=-1 / 3$, they have obtained the successful relations

$$
m_{u} \simeq \frac{3}{2} \frac{m_{e}}{m_{\tau}} \frac{\kappa}{\lambda} m_{0}, \quad m_{c} \simeq 2 \frac{m_{\mu}}{m_{\tau}} \frac{\kappa}{\lambda} m_{0}, \quad m_{t} \simeq \frac{1}{\sqrt{3}} m_{0} .
$$

As we noted in (1.3), the third quark mass does not have the suppression factor $\kappa / \lambda$. The ratio of $\kappa / \lambda$ is fixed as $\kappa / \lambda \simeq 0.02$ from the observed ratio of $m_{c} / m_{t}$. For down quark sectors, by choosing the complex parameter $b_{f}$ as $b_{d} \simeq-e^{i \pi / 10}$, they have obtained reasonable results of quark masses (not only $m_{i}^{d} / m_{j}^{d}$, but also $m_{i}^{u} / m_{j}^{d}$ ) and CKM matrix parameters. Hereafter, we will refer this model as Model I.

Therefore, our next interest is whether Model I is applicable to neutrino phenomenology under the same parameter values or not. Although they have taken $\kappa=10$ tentatively, the parameter $\kappa$ is essentially free because we does not yet observe the right-handed weak boson. [The case with a small $\kappa$ (e.g., $\sim 10$ ) is very attractive from the phenomenological standpoint, because the case can bring us detectable new physics in abundance [9]. However, in the present paper, since we intend to give a unified description of quark and neutrino mass matrices without assuming further additional intermediate mass scale, we do not consider the case with $\kappa \sim 10$.] Also, although the parameters $b_{f}$ have been taken as $b_{e}=0$, $b_{u}=-1 / 3$ and $b_{d} \simeq-e^{i \pi / 10}$ in Ref. [5], the value of $b_{\nu}$ in the neutrino sector is still free. Therefore, for the neutrino masses and mixings, we have three adjustable parameters, i.e., $b_{\nu}$ (complex) and $m_{0} / \lambda$ (real). 
For typical values of $b_{\nu}, b_{\nu} \simeq-1 / 3, b_{\nu} \simeq-1 / 2$ and $b_{\nu} \simeq-1$, the masses $m_{i}^{\nu}$ $(i=1,2,3)$ and mixings $U_{\alpha i}(\alpha=e, \mu, \tau ; i=1,2,3)$ for three light neutrinos are given as follows [10].

Case $b_{\nu}=-1 / 3+\Delta b_{\nu} \quad\left(1 \gg\left|\Delta b_{\nu}\right| \neq 0\right)$ :

$$
\begin{gathered}
m_{1}^{\nu} \simeq \frac{3}{2} \frac{m_{e}}{m_{\tau}} \frac{m_{0}}{\lambda}, \quad m_{2}^{\nu} \simeq 2 \frac{m_{\mu}}{m_{\tau}} \frac{m_{0}}{\lambda}, \quad m_{3}^{\nu} \simeq \frac{2 \sqrt{2}}{27\left|\Delta b_{\nu}\right|} \frac{m_{0}}{\lambda}, \\
U \simeq\left(\begin{array}{ccc}
1 & \frac{1}{2} \sqrt{\frac{m_{e}}{m_{\mu}}} & \sqrt{\frac{m_{e}}{m_{\tau}}} \\
-\frac{1}{2} \sqrt{\frac{m_{e}}{m_{\mu}}} & 1 & \sqrt{\frac{m_{\mu}}{m_{\tau}}} \\
-\frac{1}{2} \sqrt{\frac{m_{e}}{m_{\tau}}} & -\sqrt{\frac{m_{\mu}}{m_{\tau}}} & 1
\end{array}\right),
\end{gathered}
$$

Case $b_{\nu} \simeq-1 / 2$ :

$$
\begin{gathered}
m_{1}^{\nu} \simeq 2 \frac{m_{e}}{m_{\tau}} \frac{m_{0}}{\lambda}, \quad m_{2}^{\nu} \simeq m_{3}^{\nu} \simeq \sqrt{\frac{m_{\mu}}{m_{\tau}}} \frac{m_{0}}{\lambda} \\
U \simeq\left(\begin{array}{ccc}
1 & \sqrt{\frac{m_{e}}{2 m_{\mu}}} & \sqrt{\frac{m_{e}}{2 m_{\mu}}} \\
-\sqrt{\frac{m_{e}}{m_{\mu}}} & \frac{1}{\sqrt{2}} & \mp \frac{1}{\sqrt{2}} \\
-\sqrt{\frac{m_{e}}{m_{\tau}}} & \pm \frac{1}{\sqrt{2}} & \frac{1}{\sqrt{2}}
\end{array}\right),
\end{gathered}
$$

Case $b_{\nu} \simeq-1$ :

$$
m_{1}^{\nu} \simeq m_{2}^{\nu} \simeq \sqrt{\frac{m_{e} m_{\mu}}{m_{\tau}^{2}}} \frac{m_{0}}{\lambda}, \quad m_{3}^{\nu} \simeq \frac{1}{2} \frac{m_{0}}{\lambda}
$$




$$
U \simeq\left(\begin{array}{ccc}
\frac{1}{\sqrt{2}} & \mp \frac{1}{\sqrt{2}} & -\sqrt{\frac{m_{e}}{m_{\tau}}} \\
\pm \frac{1}{\sqrt{2}} & \frac{1}{\sqrt{2}} & -\sqrt{\frac{m_{\mu}}{m_{\tau}}} \\
\sqrt{\frac{m_{\mu}}{2 m_{\tau}}} & \sqrt{\frac{m_{\mu}}{2 m_{\tau}}} & 1
\end{array}\right) .
$$

\section{Neutrino Data and their Interpretations}

As possible evidences for non-zero neutrino masses, at present, the following data are known:

(a) The solar neutrino data [11] with the Mikheyev-Smirnov-Wolfenstein (MSW) effect [12] have suggested

$$
\Delta m_{\odot}^{2} \simeq 6 \times 10^{-6} \mathrm{eV}^{2}, \quad \sin ^{2} 2 \theta_{\odot} \simeq 7 \times 10^{-3}
$$

(the small-angle solution), or

$$
\Delta m_{\odot}^{2} \simeq 10^{-5} \mathrm{eV}^{2}, \quad \sin ^{2} 2 \theta_{\odot} \simeq 0.8
$$

(the large-angle solution).

(b) The atmospheric neutrino data reported by the Kamiokande collaboration [13] have suggested a neutrino mixing $\nu_{\mu} \leftrightarrow \mu_{x}$ :

$$
\Delta m_{a t m}^{2} \simeq 1.6(1.8) \times 10^{-2} \mathrm{eV}^{2}, \quad \sin ^{2} 2 \theta_{a t m} \simeq 1
$$

for $x=\mu(x=e)$.

(c) The neutrino oscillation $\left(\bar{\nu}_{\mu} \rightarrow \bar{\nu}_{e}\right)$ experiment by the liquid scintillator neutrino detector (LSND) [14] at Los Alamos has been reported nonzero neutrino mass:

$$
\left(\Delta m^{2}, \sin ^{2} 2 \theta\right)_{L S N D} \simeq\left(0.3 \mathrm{eV}^{2}, 0.04\right)-\left(2 \mathrm{eV}^{2}, 0.002\right)
$$

(d) A cosmological model with cold+hot dark matter (CHDM) suggests [15]

$$
m_{1}^{\nu}+m_{2}^{\nu}+m_{3}^{\nu} \simeq 4.8 \mathrm{eV}
$$

These mass values and mixings (4.1)-(4.3) and (4.5) are not based on direct observations of masses and mixings and they are highly model-dependent. On the other hand, the experiment which reported the result (4.4) is still controversial [16]. (For 
convenience, hereafter, we will refer these data $(4.1)-(4.5)$ as $\nu_{\odot}^{\text {small }}, \nu_{\odot}^{\text {large }}, \nu_{\text {atm }}$, $\nu_{L S N D}$ and $\nu_{C H D M}$, respectively.)

Since $\Delta m_{L S N D}^{2} \gg \Delta m_{\text {atm }}^{2} \gg \Delta m_{\odot}^{2}$, we investigate only the following two cases: (A) $\Delta m_{32}^{2} \gg \Delta m_{21}^{2} \quad$ (i.e., $b_{\nu} \simeq-1$ and $b_{\nu} \simeq-1 / 3$ ); (B) $\Delta m_{32}^{2} \ll \Delta m_{21}^{2}$ (i.e., $b_{\nu} \simeq-1 / 2$ ) , where $\Delta m_{i j}^{2}=m_{i}^{\nu 2}-m_{j}^{\nu 2}$. Of course, in the three family model, we cannot assign the three values $\Delta m_{L S N D}^{2}, \Delta m_{\text {atm }}^{2}$ and $\Delta m_{\odot}^{2}$ to $\left(\Delta m_{32}^{2}, \Delta m_{21}^{2}\right)$ simultaneously. For the case $(\mathrm{A})$, we consider two cases: $\left(\mathrm{A}_{1}\right)\left(\Delta m_{32}^{2}, \Delta m_{21}^{2}\right)=$ $\left(\Delta m_{L S N D}^{2}, \Delta m_{\odot}^{2}\right)$ and $\left(\mathrm{A}_{2}\right)\left(\Delta m_{32}^{2}, \Delta m_{21}^{2}\right)=\left(\Delta m_{L S N D}^{2}, \Delta m_{\text {atm }}^{2}\right)$. Similarly, for the case (B), we consider two cases: $\left(\mathrm{B}_{1}\right)\left(\Delta m_{32}^{2}, \Delta m_{21}^{2}\right)=\left(\Delta m_{\odot}^{2}, \Delta_{L S N D}^{2}\right)$ and $\left(\Delta m_{32}^{2}, \Delta m_{21}^{2}\right)=\left(\Delta m_{a t m}^{2}, \Delta m_{L S N D}^{2}\right)$. We do not consider the cases $\left(\Delta m_{a t m}^{2}, \Delta m_{\odot}^{2}\right)$ and $\left(\Delta m_{\odot}^{2}, \Delta m_{\text {atm }}^{2}\right)$, because it is readily known that the cases cannot give the observed large mixing $\sin ^{2} 2 \theta_{\text {atm }} \sim 1$ in $\nu_{\text {atm }}$.

In the three-family model, the neutrino oscillation $P\left(\nu_{\alpha} \rightarrow \nu_{\beta}\right)(\alpha \neq \beta)$ is given by

$$
P\left(\nu_{\alpha} \rightarrow \nu_{\beta}\right)=S_{21}^{\alpha \beta} S_{21}+S_{31}^{\alpha \beta} S_{31}+S_{32}^{\alpha \beta} S_{32}
$$

where

$$
\begin{gathered}
S_{i j}^{\alpha \beta}=-4 \Re\left(U_{\alpha i} U_{\beta i}^{*} U_{\alpha j}^{*} U_{\beta j}\right), \\
S_{i j}=\sin ^{2}\left(L \Delta m_{i j}^{2} / 4 E_{\nu}\right),
\end{gathered}
$$

and we have neglected $C P$-violation terms in (4.6).

In the case $\left(\mathrm{A}_{1}\right)$, since $\Delta m_{32}^{2} \gg \Delta m_{a t m}^{2} \gg \Delta m_{21}^{2}$, we can regard $S_{i j}$ as $S_{21} \simeq 0$ and $\left\langle S_{31}\right\rangle=\left\langle S_{32}\right\rangle=1 / 2$ for the atmospheric neutrinos, where $\left\langle S_{i j}\right\rangle$ denotes the mean value of $S_{i j}\left(L / E_{\nu}\right)$. Then, by using $P\left(\nu_{\alpha} \rightarrow \nu_{\beta}\right) \simeq 2\left|U_{\alpha 3}\right|^{2}\left|U_{\beta 3}\right|^{2}$, the ratio

$$
R_{a t m}=\frac{\left(\nu_{\mu} / \nu_{e}\right)_{d a t a}}{\left(\nu_{\mu} / \nu_{e}\right)_{M C}} \simeq \frac{P\left(\nu_{\mu} \rightarrow \nu_{\mu}\right)+\frac{1}{2} P\left(\nu_{e} \rightarrow \nu_{\mu}\right)}{P\left(\nu_{e} \rightarrow \nu_{e}\right)+2 P\left(\nu_{\mu} \rightarrow \nu_{e}\right)}
$$

is expressed as

$$
R_{a t m} \simeq \frac{1-\left|U_{e 3}\right|^{2}\left|U_{\mu 3}\right|^{2}-2\left|U_{\mu 3}\right|^{2}\left|U_{\tau 3}\right|^{2}}{1+2\left|U_{e 3}\right|^{2}\left|U_{\mu 3}\right|^{2}-2\left|U_{e 3}\right|^{2}\left|U_{\tau 3}\right|^{2}}
$$

Therefore, in the case $\left(A_{1}\right)$, instead of (4.3), we temporize with (4.10) whose value is consistent with the observed value [13]

$$
R_{a t m}=0.57_{-0.07}^{+0.08} \pm 0.07
$$

However, both cases $b_{\nu} \simeq-1 / 3$ and $b_{\nu} \simeq-1$, from (3.7) and (3.11), respectively, give $R_{a t m} \simeq 1-2 m_{\mu} / m_{\tau} \simeq 1$, which is in disagreement with the observed value 
(4.11). On the other hand, the mixing parameter $\sin ^{2} 2 \theta_{\odot}$ in the MSW solutions is interpreted by

$$
\sin ^{2} 2 \theta_{\odot}=4\left|U_{e 1}\right|^{2}\left|U_{e 2}\right|^{2}
$$

The cases $b_{\nu} \simeq-1 / 3$ and $b_{\nu} \simeq-1$ give

$$
\sin ^{2} 2 \theta_{\odot} \simeq m_{e} / m_{\mu} \simeq 0.005
$$

and

$$
\sin ^{2} 2 \theta_{\odot} \simeq 1
$$

which are consistent with the small- and large-angle solutions (4.1) and (4.2), respectively.

In the case $\left(\mathrm{A}_{2}\right)$, since $\Delta m_{\odot}^{2} \ll \Delta m_{21}^{2} \ll \Delta m_{32}^{2}$, we can regard $S_{i j}$ as $\left\langle S_{21}\right\rangle=$ $\left\langle S_{31}\right\rangle=\left\langle S_{32}\right\rangle=1 / 2$ for $\nu_{\odot}$. Then, we obtain

$$
P_{e e} \equiv P\left(\nu_{e} \rightarrow \nu_{e}\right)=\left|U_{e 1}\right|^{4}+\left|U_{e 2}\right|^{4}+\left|U_{e 3}\right|^{4} .
$$

According to the recent analysis by Acker and Pakvasa [17] we will search for a solution which is consistent with

$$
P_{e e}=0.42-0.52
$$

The cases $b_{\nu} \simeq-1 / 3$ and $b_{\nu} \simeq-1$, from (3.7) and (3.11), give $P_{e e} \simeq 1$ and $P_{e e} \simeq 1 / 2$, respectively. Therefore, only the case $b_{\nu} \simeq-1$ can explain the solar neutrino data. On the other hand, the mixing parameter $\sin ^{2} 2 \theta_{a t m}$ is interpreted by

$$
\sin ^{2} 2 \theta_{a t m}=S_{21}^{\mu e}=-4 \Re\left(U_{\mu 2} U_{e 2}^{*} U_{\mu 1}^{*} U_{e 1}\right) .
$$

The cases $b_{\nu} \simeq-1 / 3$ and $b_{\nu} \simeq-1$ give $\sin ^{2} 2 \theta_{\text {atm }} \simeq m_{e} / m_{\mu} \simeq 0.005$ and $\sin ^{2} 2 \theta_{\text {atm }} \simeq$ 1 , respectively. Again, only the case $b_{\nu} \simeq-1$ is favorable to the data.

In both cases $\left(\mathrm{A}_{1}\right)$ and $\left(\mathrm{A}_{2}\right)$, the mixing parameter $\sin ^{2} 2 \theta_{L S N D}$ is given by

$$
\sin ^{2} 2 \theta_{L S N D}=4\left|U_{e 3}\right|^{2}\left|U_{\mu 3}\right|^{2} \text {. }
$$

The cases $b_{\nu} \simeq-1 / 3$ and $b_{\nu} \simeq-1$ give $\sin ^{2} 2 \theta_{L S N D} \simeq 4 m_{e} m_{\mu} / m_{\tau}^{2} \simeq 7 \times 10^{-5}$, which is too small compared with the observed mixing value (4.4).

Similarly, for the case $\left(\mathrm{B}_{1}\right)$, we use the expressions

$$
\begin{gathered}
\sin ^{2} 2 \theta_{\odot}=4\left|U_{e 3}\right|^{2}\left|U_{e 2}\right|^{2} . \\
R_{a t m} \simeq \frac{1-\left|U_{e 1}\right|^{2}\left|U_{\mu 1}\right|^{2}-2\left|U_{\mu 1}\right|^{2}\left|U_{e 1}\right|^{2}}{1+2\left|U_{e 1}\right|^{2}\left|U_{\mu 1}\right|^{2}-2\left|U_{e 1}\right|^{2}\left|U_{\tau 1}\right|^{2}} .
\end{gathered}
$$


The mixing matrix (3.9) gives $\sin ^{2} 2 \theta_{\odot} \simeq\left(m_{e} / m_{\mu}\right)^{2} \simeq 2 \times 10^{-5}$ and $R_{a t m} \simeq 1-$ $m_{e} / 2 m_{\mu} \simeq 1$, which are in disagreement with the data.

In the case $\left(\mathrm{B}_{2}\right)$, the solar neutrino data is explained by $P_{e e}$ given by $(4.15)$, and the mixing parameter in $\nu_{a t m}$ is expressed by

$$
\sin ^{2} 2 \theta_{a t m}=S_{23}^{\mu \tau}=-4 R e\left(U_{\mu 2} U_{\tau 2}^{*} U_{\mu 3}^{*} U_{\tau 3}\right)
$$

Since the mixing matrix (3.9) gives $P_{e e} \simeq 1$ and $\sin ^{2} 2 \theta_{a t m} \simeq 1$, we fail to explain the $\nu_{\odot}$ data, but we can understand the large mixing in $\nu_{a t m}$.

For both cases $\left(B_{1}\right)$ and $\left(B_{2}\right)$, the mixing parameter $\sin ^{2} 2 \theta_{L S N D}$ is given by

$$
\sin ^{2} 2 \theta_{L S N D}=4\left|U_{e 1}\right|^{2}\left|U_{\mu 1}\right|^{2} \simeq 4 m_{e} / m_{\mu} \simeq 0.02
$$

where we have used the mixing matrix (3.9) for $b_{\nu} \simeq-1 / 2$. The mixing value (4.22) corresponds to $\Delta m_{L S N D}^{2} \simeq 0.5 \mathrm{eV}^{2}$ in the LSND allowed region (4.4).

The results are summarized in Table I. We find that the present model cannot give a simultaneous interpretation for the three neutrino data, $\nu_{\odot}, \nu_{a t m}$ and $\nu_{L S N D}$. We must give up the explanation of one of the three data.

As suggested by the case $\left(\mathrm{B}_{2}\right)$ in Table $\mathrm{I}$, if we give up explaining the solar neutrino data, we can find an interesting solution: $\left(\Delta m_{32}^{2}, \Delta m_{21}^{2}\right)=\left(\Delta m_{a t m}^{2}, \Delta m_{L S N D}^{2}\right)$ with $b_{\nu} \simeq-1 / 2$. For example, the solution $b_{\nu}=-(1 / 2) e^{i \beta_{\nu}}$ with $b_{\nu}=0.12^{\circ}$ gives

$$
\begin{gathered}
\sin ^{2} 2 \theta_{a t m}=0.995, \quad \sin ^{2} 2 \theta_{L S N D}=0.0191 \\
m_{1}^{\nu}=0.000540 m_{0} / \lambda \\
m_{2}^{\nu}=0.2288 m_{0} / \lambda \\
m_{3}^{\nu}=0.2326 m_{0} / \lambda \\
r \equiv \Delta m_{32}^{2} / \Delta m_{21}^{2}=0.0331
\end{gathered}
$$

The input value $\Delta m_{32}^{2}=\Delta m_{a t m}^{2}=0.016 \mathrm{eV}^{2}$ predicts

$$
\begin{gathered}
m_{1}^{\nu}=0.0016 \mathrm{eV}, m_{2}^{\nu}=0.695 \mathrm{eV}, \quad m_{3}^{\nu}=0.707 \mathrm{eV}, \\
\Delta m_{21}^{2}=0.483 \mathrm{eV}^{2},
\end{gathered}
$$

together with

$$
m_{0} / \lambda=3.04 \mathrm{eV}
$$

The values (4.23) and (4.27) are in good agreement with the observed SLND solution $\left(\Delta m^{2}, \sin ^{2} 2 \theta\right)_{L S N D} \simeq\left(0.5 \mathrm{eV}^{2}, 0.02\right)$. Although the sum $\sum m_{i}^{\nu}=1.4 \mathrm{eV}$ is 
somewhat small compared with the mass value in the CHDM scenario (4.5), these neutrinos can still be one of the dark matter objects

When the LSND data are neglected, the case $\left(\mathrm{A}_{2}\right)$ with $b_{\nu} \simeq-1$ is also interesting. For example, the parameter value $b_{\nu}=-e^{i \beta_{\nu}}$ with $\beta_{\nu}=1.8^{\circ}$ gives

$$
P_{e e}=0.52, \quad \sin ^{2} 2 \theta_{a t m}=0.90
$$

which are favorable to the data of $\nu_{\odot}$ and $\nu_{a t m}$. On the other hand, the input

$$
\Delta m_{21}^{2}=1.283 \times 10^{-5}\left(m_{0} / \lambda\right)^{2}=\Delta m_{a t m}^{2}=1.8 \times 10^{-2} \mathrm{eV}^{2}
$$

leads to

$$
\begin{aligned}
& m_{1}^{\nu}=0.00305 m_{0} / \lambda=0.114 \mathrm{eV}, \\
& m_{2}^{\nu}=0.00471 m_{0} / \lambda=0.176 \mathrm{eV}, \\
& m_{3}^{\nu}=0.5002 m_{0} / \lambda=18.7 \mathrm{eV},
\end{aligned}
$$

together with

$$
m_{0} / \lambda=37.5 \mathrm{eV}
$$

The value $\sum m_{i}^{\nu} \simeq 19 \mathrm{eV}$ is somewhat large to identify these neutrinos as those in the dark matter scenario. In the present case, although the third neutrino has a considerably large value $m_{3}^{\nu}=18.7 \mathrm{eV}$, the effective electron neutrino mass $\left\langle m_{\nu}\right\rangle$ is safely small compared with the upper bound on $\left\langle m_{\nu}\right\rangle$ from the neutrinoless double beta decays, $\left\langle m_{\nu}\right\rangle<0.68 \mathrm{eV}[18]$, because of the smallness of $\left|U_{e 3}^{2}\right|$, i.e.,

$$
\left\langle m_{\nu}\right\rangle=\left|m_{1}^{\nu} U_{e 1}^{2}+m_{2}^{\nu} U_{e 2}^{2}+m_{3}^{\nu} U_{e 3}^{2}\right|=0.0027 \mathrm{eV} .
$$

In these examples, we have chosen the solutions with $\beta_{\nu} \neq 0$. However, this is not essential for the numerical results. The similar results to the cases $b_{\nu}=-(1 / 2) \exp \left[i 0.12^{\circ}\right]$ and $b_{\nu}=-\exp \left[i 1.8^{\circ}\right]$ can also be obtained by choosing $b_{\nu}=-0.50004$ and $b_{\nu}=-0.97$. However, since we have taken the rational solutions of $\left|b_{f}\right|$ in the quark and charged lepton sectors, i.e., $\left|b_{u}\right|=1 / 3,\left|b_{d}\right|=1$ and $\left|b_{e}\right|=0$ [5], we have taken the rational solutions of $\left|b_{\nu}\right|$ in Table II.

In Table II, we summarize these typical cases. For reference, in Table II, we show the values of $\sin ^{2} 2 \theta^{\mu \tau}$ which is given by $\sin ^{2} 2 \theta^{\mu \tau}=4\left|U_{\mu 3}\right|^{2}\left|U_{\tau 3}\right|^{2}$ for the case $\Delta m_{32}^{2} \gg \Delta m_{21}^{2}$ and by $\sin ^{2} 2 \theta^{\mu \tau}=4\left|U_{\mu 1}\right|^{2}\left|U_{\tau 1}\right|^{2}$ for the case $\Delta m_{32}^{2} \ll \Delta m_{21}^{2}$, because CHORUS [19] and NOMAD [20] experiments at CERN are expected to present results on the $\nu_{\mu} \rightarrow \nu_{\tau}$ oscillation in the very near future. As pointed out by 
Tanimoto [21], the values of $\sin ^{2} 2 \theta^{\mu \tau}$ are very small for the case $m_{1}^{\nu} \ll m_{2}^{\nu} \simeq m_{3}^{\nu}$, i.e., $\sin ^{2} 2 \theta^{\mu \tau} \simeq 4 m_{e} / m_{\tau}$ for $b_{\nu} \simeq-1 / 2$, so that it will be hopeless to observe the $\nu_{\mu} \rightarrow \nu_{\tau}$ oscillation at CHORUS and NOMAD. The case $\Delta m_{32}^{2} \gg \Delta m_{21}^{2}$, i.e., $b_{\nu} \simeq-1$, gives a sizable value $\sin ^{2} 2 \theta^{\mu \tau} \simeq 4 m_{\mu} / m_{\tau}$, so that the case will be hopeful to observe the evidence of the $\nu_{\mu} \rightarrow \nu_{\tau}$ oscillation.

In Table II, we also list the value of the intermediate mass scales $\kappa m_{0}$ and $\lambda m_{0}$, which are obtained by the following relations:

$$
m_{t} \simeq \frac{1}{\sqrt{3}} m_{0} \simeq 180 \mathrm{GeV} \quad\left(\text { at } \mu=m_{Z}\right),
$$

from (3.5) and

$$
(\kappa / \lambda) m_{0}=m_{\tau}+m_{\mu}+m_{e}=1.850 \mathrm{GeV}, \quad\left(\text { at } \mu=m_{Z}\right)
$$

from $M_{e} \simeq(\kappa / \lambda) m_{0} Z \cdot \mathbf{1} \cdot Z$, i.e.,

$$
\begin{gathered}
m_{0} \simeq \sqrt{3} m_{t} \simeq 312 \mathrm{GeV} \quad\left(\text { at } \mu=m_{Z}\right) \\
\kappa / \lambda=5.93 \times 10^{-3} .
\end{gathered}
$$

\section{Conclusions}

In conclusion, we have investigated a universal seesaw mass matrix model with a form (1.1), and have found that the model has a possibility of giving a unified description of a mass hierarchy of quarks and leptons by assuming $\operatorname{det} M_{F}=0$ for the heavy fermion mass matrix $M_{F}$ in the up-quark sector. Here, the "hierarchy" discussed in the present paper means the mass hierarchy among the three groups, (i) top quark, (ii) quarks except for top and charged leptons, and (iii) neutrinos. For example, in Model I, the hierarchy among $m_{e}, m_{\mu}$ and $m_{\tau}$ are given by hand, i.e., by adjusting the parameters $z_{i}$ in the order-one matrices $Z \equiv Z_{L}=Z_{R}$. It is our task at the next step to answer why these matrices $Z_{L}, Z_{R}$ and $Y_{f}$ take such structures.

In the $\mathrm{SU}(2)_{L} \times \mathrm{SU}(2)_{R} \times \mathrm{U}(1)_{Y}$ model, it is likely that the neutral heavy leptons acquire Majorana masses $M_{M}$ together with the Dirac masses $M_{N}$ with the same structure and the same magnitude, i.e., $M_{M}=M_{N}$. Then, masses of the twelve Majorana neutrinos are given by (2.7)-(2.9). In the present model, heavy neutrinos with masses of the order of $\left(\kappa^{2} / \lambda\right) m_{0}$ do not appear, and, instead, pseudo-Dirac neutrinos with masses of the order of $\kappa m_{0}$ appear. 
A suitable choice of the matrices $Z_{L}, Z_{R}$ and $Y_{f}$ will give a unified descriptions of masses and mixings of quarks and leptons. As a simple example of $Z_{L}$, $Z_{R}$ and $Y_{f}$, a model with (3.1)-(3.3), Model I, has been investigated. The model can give a unified description of masses and mixings of quarks and charged leptons [5]. However, the straightforward application to the neutrino phenomenology fails to give the simultaneous explanation of the solar, atmospheric and LSND neutrino data. If we give up explaining one of these three data, for example, if we give up the explanation of the solar neutrino data, we obtain the intermediate mass scales $\kappa m_{0} \sim 10^{11} \mathrm{GeV}$ and $\lambda m_{0} \sim 10^{13} \mathrm{GeV}$ together with $m_{0}=312 \mathrm{GeV}$. Note that in spite of the largeness of $O\left(m_{R}\right)$, the model can give a reasonable value of the top quark mass $m_{t}$. (In other words, the above intermediate mass scales have estimated from the input value $m_{t}\left(m_{Z}\right)=180 \mathrm{GeV}$.)

If we take all of the neutrino data, $\nu_{\odot}, \nu_{a t m}$ and $\nu_{L S N D}$ seriously, we must seek another set of the matrices $Z_{L}, Z_{R}$ and $Y_{f}$ or we must abandon the idea $O\left(M_{N}\right)=O\left(M_{F}\right)(F=E, U, D)$. Although the model with $Z_{L}=Z_{R}$, Model I, has successfully described the quark masses and their mixings in terms of charged lepton masses, there is no reason that we should consider $Z_{L}=Z_{R}$. It will be worth while investigating a model with $Z_{L} \neq Z_{R}$ for quarks and leptons. It will also be worth while investigating a model with $O\left(M_{N}\right) \gg O\left(M_{F}\right)(F=E, U, D)$. The latter possibility will bring abundant light neutrinos into the model (for example, see a model given in [10]), although it is not economical because it bring additional intermediate mass scale into the model.

Model I discussed in Secs. III and IV is only an example. There will be many interesting versions of the universal seesaw model. The universal seesaw model will be one of the most promising models of the quark and lepton unification.

\section{Acknowledgments}

The author would like to thank M. Tanimoto for his helpful comments and calling his attention to CHORUS and NOMAD experiments. He also thank N. Muto for numerical check of the approximate expressions (2.7)-(2.9) of the $12 \times 12$ would-be seesaw mass matrix (2.1) by his computer program with high accuracy. This work was supported by the Grant-in-Aid for Scientific Research, the Ministry of Education, Science and Culture, Japan (No.08640386).

\section{References}

[ 1 ] M. Gell-Mann, P. Rammond and R. Slansky, in Supergravity, edited by P. van Nieuwenhuizen and D. Z. Freedman (North-Holland, 1979); T. Yanagida, in 
Proc. Workshop of the Unified Theory and Baryon Number in the Universe, edited by A. Sawada and A. Sugamoto (KEK, 1979); R. Mohapatra and G. Senjanovic, Phys. Rev. Lett. 44, 912 (1980).

[ 2 ] Z. G. Berezhiani, Phys. Lett. 129B, 99 (1983); Phys. Lett. 150B, 177 (1985); D. Chang and R. N. Mohapatra, Phys. Rev. Lett. 58,1600 (1987); A. Davidson and K. C. Wali, Phys. Rev. Lett. 59, 393 (1987); S. Rajpoot, Mod. Phys. Lett. A2, 307 (1987); Phys. Lett. 191B, 122 (1987); Phys. Rev. D36, 1479 (1987); K. B. Babu and R. N. Mohapatra, Phys. Rev. Lett. 62, 1079 (1989); Phys. Rev. D41, 1286 (1990); S. Ranfone, Phys. Rev. D42, 3819 (1990); A. Davidson, S. Ranfone and K. C. Wali, Phys. Rev. D41, 208 (1990); I. Sogami and T. Shinohara, Prog. Theor. Phys. 66, 1031 (1991); Phys. Rev. D47, 2905 (1993); Z. G. Berezhiani and R. Rattazzi, Phys. Lett. B279, 124 (1992); P. Cho, Phys. Rev. D48, 5331 (1994); A. Davidson, L. Michel, M. L, Sage and K. C. Wali, Phys. Rev. D49, 1378 (1994); W. A. Ponce, A. Zepeda and R. G. Lozano, Phys. Rev. D49, 4954 (1994).

[ 3 ] Z. G. Berezhiani, in Ref. [2]; A. Davidson and K. C. Wali, in Ref. [2]; S. Rajpoot, in Ref. [2]; A. Davidson, S. Ranfone and K. C. Wali, in Ref. [2]; W. A. Ponce, A. Zepeda and R. G. Lozano, in Ref. [2].

[ 4 ] CDF collaboration, F. Abe et al., Phys. Rev. Lett. 73, 225 (1994).

[ 5 ] Y. Koide and H. Fusaoka, Z. Phys. C71, 459 (1966); Prog. Theor. Phys. 97, 459 (1997).

[ 6 ] T. Morozumi, T. Satou, M. N. Rebelo and M. Tanimoto, Preprint HUPD-9704 (1977), hep-ph/9703249.

[ 7 ] L. Wolfenstein, Nucl. Phys. B185, 147 (1981); S. T. Petcov, Phys. Lett. 110B, 245 (1982); C. N. Leung and S. T. Petcov, Nucl. Phys. 125B, 461 (1983); M. Doi, M. Kenmoku, T. Kotani, H. Nishiura and E. Takasugi, Prog. Theor. Phys. 70, 1331 (1983): J. W. F. Valle, Phys. Rev. D27, 1672 (1983); J. W. F. Valle and M. Singer, Phys. Rev. D28, 540 (1983); D. Wyler and L. Wolfenstein, Nucl. Phys. B218, 205 (1983).

[ 8 ] N. Cabibbo, Phys. Rev. Lett. 10, 531 (1996); M. Kobayashi and T. Maskawa, Prog. Theor. Phys. 49, 652 (1973).

[ 9 ] Y. Koide, Preprint US-96-09 (1996), hep-ph/9701261 (unpublished); US-97-04 (1997), hep-ph/9706277, to be published in Phys. Rev. D56, (1997). 
[ 10 ] Y. Koide, Mod. Phys. Lett.A36, 2849 (1996).

[ 11 ] GALLEX collaboration, P. Anselmann et al, Phys. Lett. B327, 377 (1994); B357, 237 (1995); SAGE collaboration, J. N. Abdurashitov et al, Phys. Lett. B328, 234 (1994). Also see, N. Hata and P. Langacker, Phys. Rev. D50, 632 (1994); D52, 420 (1995).

[ 12 ] S. P. Mikheyev and A. Yu. Smirnov, Yad. Fiz. 42, 1441 (1985); [Sov. J. Nucl. Phys. 42, 913 (1985)]; Prog. Part. Nucl. Phys. 23, 41 (1989); L. Wolfenstein, Phys. Rev. D17, 2369 (1978); D20, 2634 (1979); T. K. Kuo and J. Pantaleon, Rev. Mod. Phys. 61, 937 (1989). Also see, A. Yu. Smirnov, D. N. Spergel and J. N. Bahcall, Phys. Rev. D49, 1389 (1994).

[ 13 ] Y. Fukuda et al., Phys. Lett. B335, 237 (1994). Also see, Soudan-2 collaboration, M.Goodman et al., Nucl. Phys. (Proc. Suppl.) B38, 337 (1995); IMB collaboration, D. Casper et al, Phys. Rev. Lett. 66, 2561 (1989); R. BeckerSzendy et al, Phys. Rev. D46, 3720 (1989).

[ 14 ] C. Athanassopoulos et al., Phys. Rev. Lett. 75, 2650 (1995); Phys. Rev. Lett. 77, 3082 (1996); nucl-ex/9706006 (1997).

[ 15 ] J. R. Primack, J. Holtzman, A. Klypin and D. O. Caldwell, Phys. Rev. Lett. 74, 2160 (1995); D. Pogosyan and A.Starobinsky, astro-ph/9502019.

[ 16 ] J. E. Hill, Phys. Rev. Lett. 75, 2654 (1995).

[ 17 ] A. Acker and S. Pakvasa, Phys. Lett. B397, 209 (1997).

[ 18 ] M. K. Moe, Nucl. Phys. (Proc. Suppl.) B38, 36 (1995): A. Balysh et al., Proc. of the Inst. Conference on High Energy Physics (ICHEP), 27th, Glashgow, Scotland, Eds, P. J. Bussey and I. G. Knowles, IOP, 1995, p.939 (hepex/9502007).

[ 19 ] M. Baldo-Ceolin, Nucl. Phys. (Proc. Suppl.) 35, 450 (1994); K. Winter, ibid. 38, 211 (1995).

[ 20 ] L. DiLella, Nucl. Phys. (Proc. Suppl.) 31, 319 (1993).

[ 21 ] M. Tanimoto, Phys. Rev. D53, 6632 (1996). 
Table I. Possible interpretations for the $\nu_{\odot}, \nu_{a t m}$ and $\nu_{L S N D}$ data. The parameter $r$ denotes $r=\Delta m_{32}^{2} / \Delta m_{21}^{2}$. The numerical results have obtained from the direct evaluation of the mass matrix (2.1) without using the approximate expressions (3.7), (3.9) and (3.11). The values with the symbols $\circ$ and $\bullet$ denote that those are favorable and unfavorable to the observed data, respectively.

\begin{tabular}{|c|c|c|}
\hline \multicolumn{2}{|c|}{$(\mathrm{A}) \Delta m_{32}^{2} \gg \Delta m_{21}^{2}$} & (B) $\Delta m_{32}^{2} \ll \Delta m_{21}^{2}$ \\
\hline$b_{\nu} \simeq-1$ & $b_{\nu} \simeq-1 / 3$ & $b_{\nu} \simeq-1 / 2$ \\
\hline \multicolumn{2}{|c|}{$\left(\mathrm{A}_{1}\right)\left(\Delta m_{32}^{2}, \Delta m_{21}^{2}\right)=\left(\Delta m_{L S N D}^{2}, \Delta m_{\odot}^{2}\right)$} & $\left(\mathrm{B}_{1}\right)=\left(\Delta m_{\odot}^{2}, \Delta m_{L S N D}^{2}\right)$ \\
\hline $\begin{array}{l}\text { ० } \sin ^{2} 2 \theta_{\odot} \simeq 1 \\
\text { - } R_{a t m} \simeq 0.9 \\
\text { - } \sin ^{2} 2 \theta_{L S N D} \simeq 5 \times 10^{-5} \\
\circ r \geq 10^{4}\end{array}$ & $\begin{array}{l}\text { ० } \sin ^{2} 2 \theta_{\odot} \simeq 0.04 \\
\text { - } R_{a t m} \simeq 1 \\
\text { - } \sin ^{2} 2 \theta_{L S N D} \simeq 6 \times 10^{-5} \\
\circ r \geq 10^{4}\end{array}$ & $\begin{array}{l}\text { - } \sin ^{2} 2 \theta_{\odot} \simeq 2 \times 10^{-5} \\
\text { - } R_{a t m} \simeq 1 \\
\circ \sin ^{2} 2 \theta_{L S N D} \simeq 0.02 \\
\circ r \leq 10^{-3}\end{array}$ \\
\hline \multicolumn{2}{|c|}{$\left(\mathrm{A}_{2}\right)\left(\Delta m_{32}^{2}, \Delta m_{21}^{2}\right)=\left(\Delta m_{L S N D}^{2}, \Delta m_{a t m}^{2}\right)$} & $\left(\mathrm{B}_{2}\right)=\left(\Delta m_{a t m}^{2}, \Delta m_{L S N D}^{2}\right)$ \\
\hline $\begin{array}{l}\circ P_{e e} \simeq 0.5 \\
\circ \sin ^{2} 2 \theta_{a t m} \simeq 1 \\
\circ \sin ^{2} 2 \theta_{L S N D} \simeq 5 \times 10^{-5} \\
\circ r \geq 10^{4}\end{array}$ & $\begin{array}{l}\text { - } P_{e e} \simeq 1 \\
\text { - } \sin ^{2} 2 \theta_{a t m} \simeq 4 \times 10^{-3} \\
\text { - } \sin ^{2} 2 \theta_{L S N D} \simeq 6 \times 10^{-5} \\
\text { ○ } r \geq 10^{4}\end{array}$ & $\begin{array}{l}-P_{e e} \simeq 1 \\
\circ \sin ^{2} 2 \theta_{a t m} \simeq 1 \\
\circ \sin ^{2} 2 \theta_{L S N D} \simeq 0.02 \\
\circ r \leq 10^{-3}\end{array}$ \\
\hline
\end{tabular}


Table II. Numerical results for the special values of $b_{\nu}$. The values with the underlines are input values. The values with the parentheses denote those which contradict with the observed values.

\begin{tabular}{|c|c|c|}
\hline \hline$b_{\nu}$ & $-\exp \left[i 1.8^{\circ}\right]$ & $-(1 / 2) \exp \left[i 0.12^{\circ}\right]$ \\
\hline$r \equiv \Delta m_{32}^{2} / \Delta m_{21}^{2}$ & $1.950 \times 10^{4}$ & 0.03310 \\
$\Delta m_{32}^{2}$ & $3.51 \times 10^{2} \mathrm{eV}^{2}$ & $\underline{0.016 \mathrm{eV}^{2}}$ \\
$\Delta m_{21}^{2}$ & $\underline{0.018 \mathrm{eV}^{2}}$ & $0.483 \mathrm{eV}^{2}$ \\
\hline$m_{0} / \lambda$ & $37.5 \mathrm{eV}$ & $3.04 \mathrm{eV}$ \\
\hline$m_{3}^{\nu}$ & $18.7 \mathrm{eV}$ & $0.707 \mathrm{eV}$ \\
$m_{2}^{\nu}$ & $0.176 \mathrm{eV}$ & $0.695 \mathrm{eV}$ \\
$m_{1}^{\nu}$ & $0.114 \mathrm{eV}$ & $0.00164 \mathrm{eV}$ \\
\hline$\sum m_{i}^{\nu}$ & $19.0 \mathrm{eV}$ & $1.40 \mathrm{eV}$ \\
$\left\langle m_{\nu}\right\rangle$ & $0.00705 \mathrm{eV}$ & $0.00267 \mathrm{eV}$ \\
\hline$P_{e e}$ & 0.523 & $(0.990)$ \\
$\sin ^{2} 2 \theta_{a t m}$ & 0.900 & 0.995 \\
$\sin ^{2} 2 \theta_{L S N D}$ & $(0.000048)$ & 0.0191 \\
$\sin ^{2} 2 \theta^{\mu \tau}$ & 0.211 & $5.4 \times 10^{-6}$ \\
\hline$m_{0}$ & $3.12 \times 10^{2} \mathrm{GeV}$ & $3.12 \times 10^{2} \mathrm{GeV}$ \\
$\kappa m_{0}$ & $1.54 \times 10^{10} \mathrm{GeV}$ & $1.90 \times 10^{11} \mathrm{GeV}$ \\
$\lambda m_{0}$ & $2.60 \times 10^{12} \mathrm{GeV}$ & $3.20 \times 10^{13} \mathrm{GeV}$ \\
\hline \hline
\end{tabular}

